\section{Protein Isolation and Purification}

\section{Getting Started}

Straightforward and cost-effective protein isolation and purification is one of the first steps in many experiments, useful in the determination of protein structure or biologic activity. Purification begins with processing the cells containing the protein of interest; these may be cells naturally producing the protein, or be a host cell expression system containing an engineered transgene for the protein. Isolation and purification methods to collect and concentrate a protein of interest include chromatography or electrophoresis, which separate on the basis of molecular size, charge, hydrophobicity, and/or affinity for an antibody, antigen, or substrate.

\section{More Advances Needed}

Mario Lebendiker directs the Protein Purification Facility at the Wolfson Centre for Applied Structural Biology, at the Hebrew University of Jerusalem in Israel. This facility that works with many different groups-including researchers, students, and biotechnology and pharmaceutical companies-but one of its main problems is protein aggregation of overexpressed proteins (mainly soluble aggregates that are difficult to see) since protein aggregation can interfere with various analytic techniques. To overcome this, developers are are trying to create protocols that can simultaneously check different strategies at the expression and purification levels.

Recent technical advances have made protein isolation and purification at the Protein Purification Facility easier and more productive. These include the recent development of better ultrafiltration systems; Ni columns with a higher capacity that allow the use of reducing agents; a very efficient and robust cell lysis system (via the Microfluidizer processor by Microfluidics in Newton, MA); and new separation techniques that mix ion and hydrophobic exchange in the same resin, allowing another dimension of purification. Lebendiker would like to see the development of additional new resins that mix different characteristics (for example,

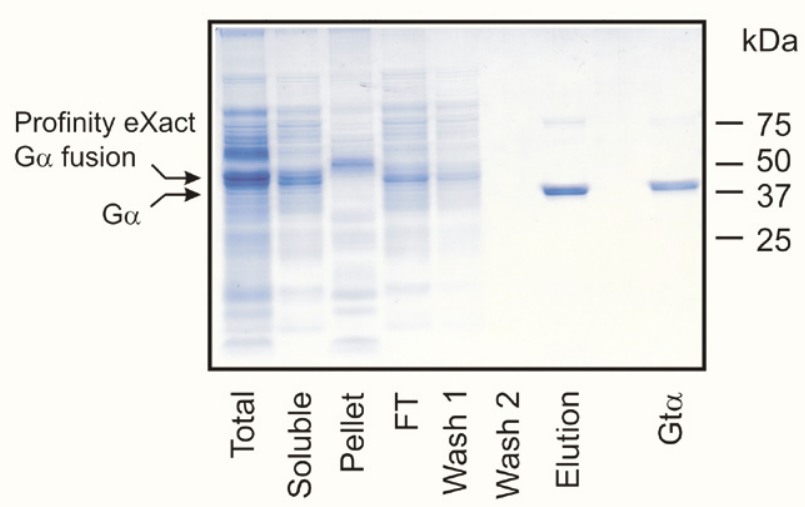

Figure 1. ChiTgel. Isolation of a Profinity eXact fusion-tagged $\mathrm{G}_{\alpha}$ chimera using the Profinia purification system. Courtesy of Kevin D. Ridge, Center for Membrane Biology, Department of Biochemistry and Molecular Biology, University of Texas Health Science Center-Houston Medical School, Houston, TX.

hydrophobic interaction chromatography with hydroxyapatite or gel filtration, or ion exchange chromatography with gel filtration or hydrophobic interaction chromatography), better ultrafiltration systems that can reduce concentration or buffer exchange time, expanded-bed adsorption systems for medium-scale laboratory use, and better endotoxin removal systems.

Lebendiker thinks that the field of membrane chromatography will be very important in the future at the industrial level, and expects to see the use of affinity chromatography, using engineered ligands tailored to specific needs.

\section{Seeing the Light}

Kevin D. Ridge of the Center for Membrane Biology, Departments of Biochemistry and Molecular Biology at the University of Texas Health Science Center at Houston, is a structural biologist who uses a commercial protein purification system to purify the membrane protein [G-protein coupled receptors (GPCRs)] for nuclear magnetic resonance (NMR) characterization. He is looking at phototransduction of the visual photoreceptor rhodopsin as a model for understanding GPCR-mediated signaling.

This work requires over-expression of soluble heterotrimeric $G$ proteins, which is difficult because of their size $(40 \mathrm{kDa})$. Using the subtilisin prodomain (Profinity eXact, Bio-Rad Laboratories, Inc.) fusion tag as part of a chimeric G-protein subunit allows purification using an immobilized, slow-cleaving mutant of the subtilisin protease. Ridge finds this system yields more soluble, functional protein than His $_{6}$-tagged protein does, and in a shorter period of time, particularly when coupled with the Profinia system for desalting.

He notes that the hydrophobic sequence of the fusion tag, designed to improve binding, helps enhance solubility of the fusion protein. He hasn't seen any effect on folding of the cleaved target protein, which allows him to look at the proteins of interest by structural techniques and to compare them biochemically and biophysically with similar proteins.

$\mathrm{He}$ is using NMR in conjunction with functional assays to investigate how the purified G-protein subunits interact during heterotrimer formation as well as how they interact with activated rhodopsin, and is beginning to investigate the biochemistry and structure of a constitutively active form of visual arrestin, which blocks GPCR binding to $\mathrm{G}$ proteins. Ridge is now looking to extend is work beyond the visual system. He also says that he's been using this technology long before it was commercialized.

\section{Transcription Factors}

One of the research interests of Harry W. Jarrett, the Lutcher Brown Distinguished Professor of Biochemistry in the Department of Chemistry at the University of Texas at San Antonio, is purification of transcription factors. $\mathrm{He}$ points out that transcription factorsproteins that bind to the promoter regions of genes, turning them on or off-are one of the most abundant types of proteins identified as a result of the human genome project. He says that there may be about 


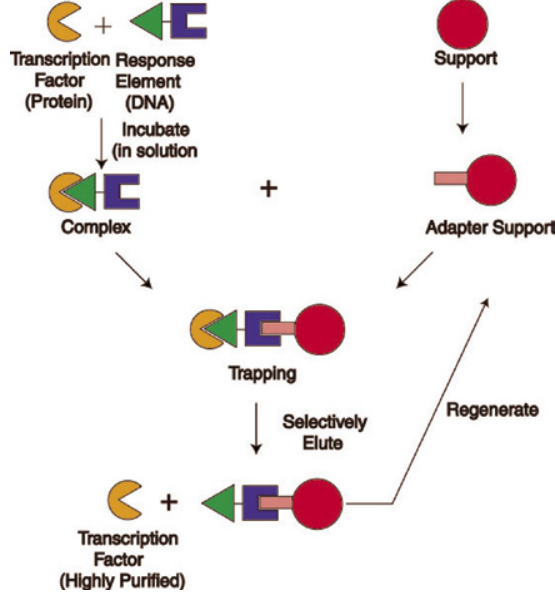

1500 transcription factors identified, but as recently as four years ago, only about 50 had been purified. His group has been working on transcription factors for over 15 years. They may be present in only 100-1000 copies per cell, although some may be more abundant. The methods that Jarrett's group used in the past, including DNA affinity chromatography, could purify proteins up to 100 -fold, which meant that in order to obtain enough protein to work with, huge volumes of cells had to be processed.

\section{"How are we ever going to understand the molecular biology of humans if it takes labs decades to characterize one promoter?"}

Jarrett's group is involved with oligonucleotide trapping, which allows purification up to several thousand-fold. This method is illustrated in Figure 1. Jarrett notes that this method has advantages over affinity chromatography in that it overcomes the nonspecific binding that transcription factors have to any DNA at micromolar concentrations. This oligonucleotide trapping occurs at nanomolar to subnanomolar concentrations at which the transcription factor should bind specifically to its response element. They are also developing other methods for transcription factor purification.

Jarrett's laboratory and collaborators are interested in looking at transcription factors that control insulin production and release and bind to the promoters of uncoupling protein 3 and c-jun, among
Figure 2. Oligo trapping. $\mathrm{CNBr}$-activated Sepharose is reacted with single stranded (AC) 5 DNA (pink rectangle) to make the adapter support. A duplex response element (green triangle) is prepared with a single stranded (GT) 5 tail (blue). This is mixed with a nuclear extract at nanomolar concentration and a specific transcription factor (orange pac-man) binds. The complex is then applied to the adapter support where the single stranded (AC)5:(GT) 5 sequences anneal, trapping the complex. The column can then be eluted in one of several different ways to yield a highly purified transcription factor. Courtesy of Harry W. Jarrett, Department of Chemistry, University of Texas San Antonio, TX.

others. He observes that although few promoters are well-characterized, that of c-jun is probably the best characterized, and yet new response elements in the promoter are still being found. He says they are starting to develop proteomic methods that could be used to purify proteins, such as removing spots identified by Southwestern blotting and preparing them for mass spectroscopy. Currently, the method does not allow for preparing protein in as large amounts as they'd like to. Jarrett's group would like to be able to identify all the proteins that bind to one promoter in one experiment, and be able to characterize them all. Roughly 30 years' worth of research has been conducted on c-jun-in several laboratories, which have generated hundreds of papers-and yet its promoter is still being characterized. "If the 23,000 human genes each have a promoter, that's 23,000 promoters. How are we ever going to understand the molecular biology of humans if it takes labs decades to characterize one promoter?" he asks.

"Wouldn't it be wonderful if we had a map of promoters in organisms? Then we could begin to understand interactions," Jarrett suggests. But, he says, until some breakthrough technology comes along, it will be difficult to answer the remaining questions. "It's not clear where [this technology] will come from. It may come out of the blue; someone will study some obscure thing and that will be the answer. Or it may come from someone methodically plodding along to improve purification techniques." The importance of transcriptions to gene function cannot be overlooked, he says. "We can't afford to leave transcription factors as a black hole."

\section{Written by Lynne Lederman, Ph.D. [II}

BioTechniques 46:87-89 (February 2009)

doi 10.2144/000113061

\section{Redefining the cell-based assay!}

\section{5 years of experience with impedance-based cell assays put into one instrument line!}

Measurements are rapid, accurate and highly reproducible. ECIS monitors cell behavior in tissue culture quantitatively and in real time. The ECIS Z \& Z $\theta$ are the new standard in life sciences for impedance-based quantification of cell behavior in vitro.

To learn more or to demo ECIS visit www.biophysics.com or call 1.866.301.ECIS (3247).

Applied
BioPhysics Quantifying Cell Behavior

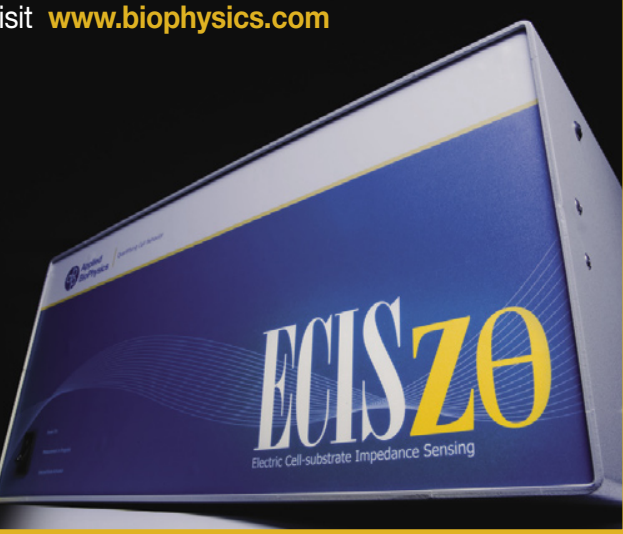

